\title{
The steady states and robustness of fuzzy discrete dynamic systems
}

\author{
Martin Gavalec $^{1}$, Ján Plavka ${ }^{2}$ \\ ${ }^{1}$ University of Hradec Králové, Rokitanského 62, 50003 Hradec Králové, Czech Republic, \\ ${ }^{2}$ Technical University of Košice, Němcovej 32, 04200 Košice, Slovakia
}

\begin{abstract}
The steady states of a fuzzy discrete dynamic system correspond to invariants (eigenvectors) of the transition matrix of the system. The structure of the eigenspace of a given fuzzy matrix is considered for various max- $\mathrm{T}$ algebras, where $\mathrm{T}$ is some triangular norm (Gödel, Łukasiewicz, product, drastic).

A given transition fuzzy matrix is called (strongly) robust if for every starting vector of a fuzzy discrete dynamic system a multiplication of a power matrix with the starting vector produces a (greatest) eigenvector of the transition matrix. A transition matrix is called weakly robust if the only possibility to arrive at an eigenvector is to start of a fuzzy discrete dynamic system by a vector that is itself an eigenvector.

We present characterizations of the eigenspace of a given transition matrix in various max- $T$ algebras. Further results concern the robustness (weak, strong robustness) of a matrix and an interval matrix (matrix with inexact data). Polynomial algorithms for checking the equivalent conditions for (weak, strong) robustness of interval fuzzy matrices are presented.
\end{abstract}

Keywords: dynamic system, eigenvector, eigenspace, robustness

\section{Introduction}

\subsection{Discrete dynamic systems}

The steady states of a complex discrete dynamic system, which is described by a given transition matrix and by fuzzy state vectors, correspond to the eigenvectors of the transition matrix. Finding all possible steady states is therefore equivalent to describing all eigenvectors. Thus, the investigation of the eigenspace structure in fuzzy algebras is important for applications, see e.g. [4], [5], [16], [22]. The eigenproblem has been studied by many authors in the case of the max-min fuzzy algebra, which is the basic one of fuzzy algebras. Interesting results have been found in describing the structure of the eigenspace. In particular, algorithms have been suggested for computing the maximal eigenvector of a given max-min matrix, see [2], [3], [6]. A generalization of the problem has also been studied in structures like semi-modules or distributive lattices [12], [24].

The structure of the eigenspace in the max-min fuzzy algebra as the union of intervals of permuted non-decreasing eigenvectors has been described in [8]. The approach from [8] can be transferred to other fuzzy algebras of type max- $\mathrm{T}$ with a triangular norm $T$. This has been done for the drastic t-norm in [10], where the structure of the eigenspace of a given max-drast fuzzy matrix is completely described. Similar results for square matrices in max-Łukasiewicz algebra (with the Łukasiewicz tnorm) have been published in [21]. The structure of the eigenspace for matrices in the max-prod algebra (with the product t-norm) is presented in [11].

\subsection{Eigenproblem in max- $T$ algebra}

Let $T$ be one of the triangular norms used in fuzzy theory, let us denote the real unit interval by $\mathcal{I}=$ $\langle 0,1\rangle$. By the max- $T$ algebra we understand the triple $(\mathcal{I}, \oplus, \otimes)$ with the binary operations $\oplus=\max$ $(x \oplus y=\max (x, y))$ and $\otimes=T$ on $\mathcal{I}$. For given natural $n$, we write $N=\{1,2, \ldots, n\}$. The notation $\mathcal{I}(n, n)(\mathcal{I}(n))$ denotes the set of all square matrices (all vectors) of a given dimension $n$ over $\mathcal{I}$. The operations $\oplus, \otimes$ are extended to matrices and vectors in the standard way.

The eigenproblem for a given matrix $A \in \mathcal{I}(n, n)$ in max-T algebra consists in finding an eigenvector $x \in \mathcal{I}(n)$ for which $A \otimes x=x$ holds true. The eigenspace of $A \in \mathcal{I}(n, n)$ is denoted by $\mathcal{F}(A)=$ $\{x \in \mathcal{I}(n) ; A \otimes x=x\}$.

Various triangular norms are used in the fuzzy sets theory. The most frequent of them are

$$
\begin{array}{ll}
\text { Gödel t-norm } & G(x, y)=\min (x, y) \\
\text { Łukasiewicz t-norm } & L(x, y)=\max (x+y-1,0) \\
\text { product t-norm } & \operatorname{prod}(x, y)=(x \cdot y)
\end{array}
$$

drastic t-norm

$$
\operatorname{drast}(x, y)= \begin{cases}\min (x, y) & \text { if } \max (x, y)=1 \\ 0 & \text { if } \max (x, y)<1\end{cases}
$$

We shall consider various types of the max$\mathrm{T}$ algebra $(\mathcal{I}, \oplus, \otimes)$ with $\oplus=\max (x, y)$ and with $\otimes$ equal to one of the binary operations $G(x, y), L(x, y), \operatorname{prod}(x, y), \operatorname{drast}(x, y)$. The investigation of the eigenspace structure can be simplified by permuting any vector $x \in \mathcal{I}(n)$ to a nondecreasing form. For given permutations $\varphi, \psi \in P_{n}$ we denote by $A_{\varphi \psi}$ the matrix with rows permuted 
by $\varphi$ and columns permuted by $\psi$, and we denote by $x_{\varphi}$ the vector permuted by $\varphi$. It can be easily shown that the following theorem holds, see [8].

Theorem 1.1 [8] Let $A \in \mathcal{I}(n, n), x \in \mathcal{I}(n)$ and $\varphi \in P_{n}$. Then $x \in \mathcal{F}(A)$ if and only if $x_{\varphi} \in$ $\mathcal{F}\left(A_{\varphi \varphi}\right)$.

We define the non-decreasing eigenspace $\mathcal{F} \leq(A)$ of a matrix $A \in \mathcal{I}(n, n)$ as

$\left\{x \in \mathcal{I}(n) ; A \otimes x=x,(\forall i, j)\left[i \leq j \Rightarrow x_{i} \leq x_{j}\right]\right\}$,

and the increasing eigenspace $\mathcal{F}^{<}(A)$ as

$\left\{x \in \mathcal{I}(n) ; A \otimes x=x,(\forall i, j)\left[i<j \Rightarrow x_{i}<x_{j}\right]\right\}$.

Analogous notation $\mathcal{I}^{\leq}(n)$ and $\mathcal{I}^{<}(n)$ will be used without condition $A \otimes x=x$. We also use notation $\mathcal{I}_{\bar{\varphi}}^{\leq}(n)=\left\{x \in \mathcal{I}(n) ; x_{\varphi} \in \mathcal{I}^{\leq}(n)\right\}, \mathcal{I}_{\varphi}^{<}(n)=\{x \in$ $\left.\mathcal{I}(n) ; x_{\varphi} \in \mathcal{I}^{<}(n)\right\}, \mathcal{F}_{\varphi}^{\leq}(A)=\left\{x \in \mathcal{F}(A) ; x_{\varphi} \in\right.$ $\mathcal{I} \leq(n)\}$ and $\mathcal{F}_{\varphi}^{<}(A)=\left\{x \in \mathcal{F}(A) ; x_{\varphi} \in \mathcal{I}^{<}(n)\right\}$, with $\varphi \in P_{n}$.

\subsection{Applications of the eigenspace in max-T algebra}

The orbit $x, A \otimes x, \ldots, A^{k} \otimes x, \ldots$, where $A^{k}=$ $A \otimes \ldots \otimes A$, represents the evolution of a discrete dynamic system. Regarding the orbits, one wishes to know the set of starting vectors from which a given objective can be achieved. One of the most natural objectives in max- $\mathrm{T}$ algebra, where the ultimate periodicity of the orbits often occurs, is to arrive at an eigenvector. The set of starting vectors from which one reaches an eigenvector (the greatest eigenvector) of $A$ after a finite number of stages, is called the attraction (strongly attraction) set of $A([20])$. In general, the attraction set contains the set of all eigenvectors, an interval vector $\boldsymbol{X}=\{x ; \underline{x} \leq x \leq \bar{x}\}$ but it can be also as big as the whole space.

This leads to the question in which case is the attraction set precisely the same as the set of all eigenvectors or the set of all eigenvectors belonging to $\boldsymbol{X}$ ? Matrices with this property are called weakly robust ([18] [19]). Furthermore, a max-T matrix is called robust if the steady-state regime of a discrete-events system is reached with any starting vector ([13], [14]), and it is called strongly robust ([17]) if the strongly attraction set is reached with any "available" starting vector. In the special case of max- $T$ algebra it can be argued that an orbit can stabilize at a fixed point $(A \otimes x=x)$, but not at an eigenvector with an eigenvalue different from unity. Therefore, by eigenvectors of $A$ we shall mean the fixed points of $A$ (satisfying $A \otimes x=x$ ).

In Sections 3, 4, 5, we consider simple and interval versions of the weak robustness, robustness and strong robustness.

\section{Eigenspace structure in various max-T algebras}

\subsection{Eigenvectors in max-min algebra}

If the binary operation $\otimes$ coincides with the minimum operation, then the increasing eigenspace $\mathcal{F}^{<}(A)$ can be described as an interval of increasing eigenvectors. In [8], the bounds $m^{\star}(A), M^{\star}(A) \in$ $\mathcal{I}(n)$ of the interval have been defined

$$
\begin{aligned}
m^{(j)}(A):=\max _{k>j} a_{j k} & M^{(j)}(A):=\max _{k \geq j} a_{j k} \\
m_{i}^{\star}(A):=\max _{j \leq i} m^{(j)}(A) & M_{i}^{\star}(A):=\min _{j \geq i} M^{(j)}(A)
\end{aligned}
$$

and the following theorem has been proved.

Theorem 2.1 [8] Let $A \in \mathcal{I}(n, n)$ and let $x \in \mathcal{I}(n)$ be an increasing vector. Then $x \in \mathcal{F}(A)$ if and only if $m^{\star}(A) \leq x \leq M^{\star}(A)$. In formal notation,

$$
\mathcal{F}^{<}(A)=\left\langle m^{\star}(A), M^{\star}(A)\right\rangle \cap \mathcal{I}^{<}(n) .
$$

An analogous description has been given in [8] for the non-decreasing eigenspace $\mathcal{F} \leq(A)$. In particular, the constant eigenvectors are characterized as follows.

Theorem 2.2 [8] Let $A \in \mathcal{I}(n, n)$ and let $x=$ $(c, c, \ldots, c) \in \mathcal{I}(n)$.Then $x \in \mathcal{F}(A)$ if and only if $0 \leq c \leq \min _{i \in N} \max _{j \in N} a_{i j}$.

Hence, in view of Theorem 1.1, the structure of $\mathcal{F}(A)$ in a max-min algebra $(\mathcal{I}, \oplus, \otimes)$ has been completely described for any $A \in \mathcal{I}(n, n)$.

The $\max -G$ fuzzy algebra with the Gödel tnorm is a special case of a max-min algebra, thus the eigenspace for this case is described by Theorems 1.1-2.2 and the analogous theorem for the non-decreasing eigenvectors.

Example 2.3 The above results are illustrated by computing the eigenspace $\mathcal{F}(A)$ of a twodimensional matrix $A \in \mathcal{I}(2,2)$. Clearly, any twodimensional vector $x=\left(x_{1}, x_{2}\right)$ is either increasing, or decreasing, or constant, thus the eigenspace can be written in the form $\mathcal{F}(A)=\mathcal{F}^{<}(A) \cup \mathcal{F}^{>}(A) \cup$ $\mathcal{F}^{=}(A)$. The decreasing eigenspace $\mathcal{F}^{>}(A)$ is computed using the permutation $\varphi=\left(\begin{array}{ll}1 & 2 \\ 2 & 1\end{array}\right)$ and the permuted matrix $A_{\varphi \varphi}$, according to Theorem 1.1.

$$
A=\left(\begin{array}{cc}
0.7 & 0.3 \\
0.2 & 1
\end{array}\right), \quad A_{\varphi \varphi}=\left(\begin{array}{cc}
1 & 0.2 \\
0.3 & 0.7
\end{array}\right) \text {. }
$$

By Theorems 2.1 and 2.2, the eigenspace $\mathcal{F}(A)$ consists of

- increasing eigenvectors with $0.3 \leq x_{1} \leq 0.7$ and $x_{1}<x_{2} \leq 1$,

- decreasing eigenvectors with $0.2 \leq x_{2} \leq 0.7$ and $x_{2}<x_{1} \leq 0.7$,

- constant eigenvectors $x=(c, c)$ with $0 \leq c \leq$ 0.7 . 

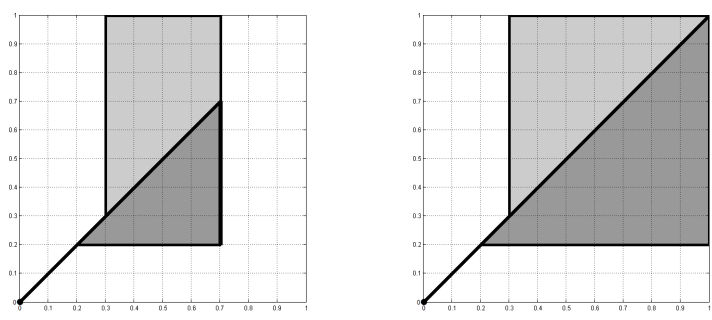

Figure 1: $\mathcal{F}(A)$ and $\mathcal{F}(B)$ in the max-G (max-min) algebra

Example 2.4 In this example we change the entry $a_{11}=0.7$ to $b_{11}=1$, and leave the remaining entries unchanged. Similarly as above, we compute the eigenspace $\mathcal{F}(B)=\mathcal{F}^{<}(B) \cup \mathcal{F}^{>}(B) \cup \mathcal{F}^{=}(B)$ of matrix $B$ using its permuted counterpart $B_{\varphi \varphi}$.

$$
B=\left(\begin{array}{cc}
1 & 0.3 \\
0.2 & 1
\end{array}\right) \quad, \quad B_{\varphi \varphi}=\left(\begin{array}{cc}
1 & 0.2 \\
0.3 & 1
\end{array}\right) \text {. }
$$

The eigenspace $\mathcal{F}(B)$ consists of

- increasing eigenvectors with $0.3 \leq x_{1} \leq 1$ and $x_{1}<x_{2} \leq 1$

- decreasing eigenvectors with $0.2 \leq x_{2} \leq 1$ and $x_{2}<x_{1} \leq 1$

- constant eigenvectors $x=(c, c)$ with $0 \leq c \leq 1$.

Eigenspaces $\mathcal{F}(A), \mathcal{F}(B)$ are shown in Figure 1 .

\subsection{Eigenvectors in max-prod algebra}

The results contained in this subsection and the detailed proofs can be found in [11]. We work in the max-prod fuzzy algebra $(\mathcal{I}, \oplus, \otimes)$ with binary operation $\oplus=\max$ and $\otimes=\operatorname{prod}$. Hence,

$$
\begin{aligned}
& (x \oplus y)_{i}=\max \left(x_{i}, y_{i}\right) \\
& (x \otimes y)_{i}=x_{i} \cdot y_{i}
\end{aligned}
$$

for every $x, y \in \mathcal{I}(n)$ and $i \in N$.

For every vector $x \in \mathcal{I}(n)$ define the quotient vector $q=q(x) \in \mathcal{I}(n)$ by

$$
q_{i}= \begin{cases}x_{i} / x_{i+1} & \text { if } i \in N \backslash\{n\}, x_{i+1} \neq 0 \\ 1 & \text { if } i \in N \backslash\{n\}, x_{i+1}=0 \\ x_{n} & \text { if } i=n\end{cases}
$$

Proposition 2.5 Suppose $x \in \mathcal{I}(n)$ and $n>1$. Then $x \in \mathcal{I}^{<}(n)$ if and only if $q=q(x)$ fulfills the following inequalities

$$
\begin{aligned}
& 0 \leq q_{1}<1 \\
& 0<q_{i}<1 \\
& 0<q_{n} \leq 1
\end{aligned} \text { for every } i \in N \backslash\{1, n\}
$$

Proposition 2.6 Suppose $A \in \mathcal{I}(n, n)$ and $x \in$ $\mathcal{I}^{<}(n)$. Then $x \in \mathcal{F}^{<}(A)$ if and only if for every $i \in N$ the following hold

$$
\begin{array}{r}
a_{i j} \leq q_{i} q_{i+1} \cdots q_{j-1} \\
\text { for every } j \in N, j>i \\
a_{i i}=1 \text { or } \quad a_{i j}=q_{i} q_{i+1} \cdots q_{j-1} \\
\text { for some } j \in N, j>i
\end{array}
$$

Theorem 2.7 [11] If $A \in \mathcal{I}(n, n)$ and $\mathcal{F}^{<}(A) \neq \emptyset$, then the following conditions are satisfied

$$
\begin{array}{r}
a_{i j}<1 \text { for all } i, j \in N, i<j, \\
a_{n n}=1 .
\end{array}
$$

Remark 2.8 It can be easily seen that in the case $n=2$ the necessary conditions in Theorem 2.7 are also sufficient.

Theorem 2.9 [11] Let $A \in \mathcal{I}(2,2) . \mathcal{F}^{<}(A) \neq \emptyset$ if and only if $a_{12}<1$ and $a_{22}=1$. If this is the case, then

$$
\begin{gathered}
\mathcal{F}^{<}(A)=\left\{\left(x_{1}, x_{2}\right) \in \mathcal{I}(2,2) ;\right. \\
\left.a_{12} x_{2} \leq x_{1}<x_{2}, 0<x_{2} \leq 1\right\}, \quad \text { if } a_{11}=1 \\
\mathcal{F}^{<}(A)=\left\{\left(x_{1}, x_{2}\right) \in \mathcal{I}(2,2) ;\right. \\
\left.a_{12} x_{2}=x_{1}, 0<x_{2} \leq 1\right\}, \text { if } a_{11}<1
\end{gathered}
$$

The set of all constant eigenvectors of a matrix $A$ is denoted by $\mathcal{F}^{=}(A)$.

Theorem 2.10 [11] Let $A \in \mathcal{I}(n, n)$. If $\max \left\{a_{i j} ; j \in N\right\}=1$ for every $i \in N$, then $\mathcal{F}^{=}(A)=\{(c, c, \ldots, c) ; c \in \mathcal{I}\}$. Otherwise, $\mathcal{F}^{=}(A)=\{(0,0, \ldots, 0)\}$.

Example 2.11 Similarly as in the previous subsection, the results are illustrated by computing the eigenspaces of two-dimensional matrices $A, B$ in (1) and (2).

By Theorems 2.9 and 2.10, the eigenspace $\mathcal{F}(A)$ consists of

- increasing eigenvectors of the form $x=$ $\left(0.3 x_{2}, x_{2}\right)$, with $0<x_{2} \leq 1$,

- no decreasing eigenvectors,

- one constant eigenvector $x=(0,0)$.

The eigenspace $\mathcal{F}(B)$ consists of

- increasing eigenvectors with $0.3 x_{2} \leq x_{1}<x_{2}$, $0<x_{2} \leq 1$,

- decreasing eigenvectors with $0.2 x_{1} \leq x_{2}<x_{1}$, $0<x_{1} \leq 1$

- constant vectors $x=(c, c)$ with $0 \leq c \leq 1$.

Eigenspaces $\mathcal{F}(A), \mathcal{F}(B)$ in max-prod algebra are shown in Figure 2.

\subsection{Eigenvectors in max-Luk algebra}

In this subsection, the above considerations will be performed for the Eukasiewicz t-norm. The details can be found in [21]. We shall work in the max-L fuzzy algebra $(\mathcal{I}, \oplus, \otimes)$ with binary operation $\oplus=$ 

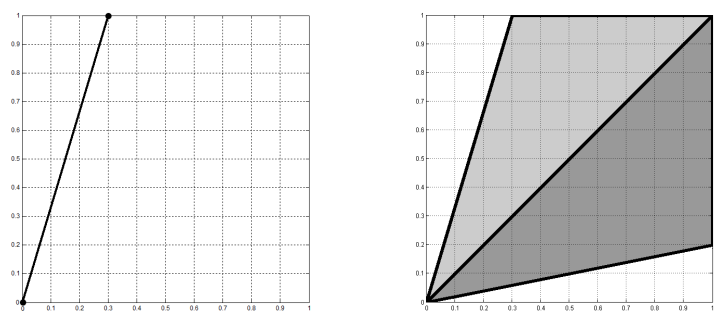

Figure 2: $\mathcal{F}(A)$ and $\mathcal{F}(B)$ in the max-prod algebra

$\max$ and $\otimes=L$. Hence, for every $x, y \in \mathcal{I}(n)$ and for every $i \in N$ we have

$$
\begin{aligned}
(x \oplus y)_{i} & =\max \left(x_{i}, y_{i}\right) \\
(x \otimes y)_{i} & =L\left(x_{i}, y_{i}\right) \\
& = \begin{cases}x_{i}+y_{i}-1 & \text { if } \min \left(x_{i}+y_{i}-1,0\right)=0 \\
0 & \text { otherwise }\end{cases}
\end{aligned}
$$

Theorem 2.12 [21] Let $A \in \mathcal{I}(n, n)$ and $x \in$ $\mathcal{I}^{<}(n)$. Then $x \in \mathcal{F}^{<}(A)$ if and only if for every $i \in N$ the following hold

$$
\begin{aligned}
& a_{i j} \leq 1+x_{i}-x_{j} \text { for every } j \in N, j \geq i, \\
& \text { if } i=1, \text { then } x_{1}=0 \text { or } a_{1 j}=1+x_{1}-x_{j} \\
& \text { for some } j \in N \\
& \text { if } i>1, \text { then } a_{i j}=1+x_{i}-x_{j} \\
& \text { for some } j \in N, j \geq i .
\end{aligned}
$$

The following theorem describes necessary conditions under which a square matrix can have a strictly increasing eigenvector.

Theorem 2.13 [21] Let $A \in \mathcal{I}(n, n)$. If $\mathcal{F}^{<}(A) \neq$ $\emptyset$ then the following conditions are satisfied

$$
\begin{aligned}
& a_{i j}<1 \text { for all } i, j \in N, i<j, \\
& a_{n n}=1 .
\end{aligned}
$$

Remark 2.14 It can be easily seen that in the case $n=2$ the necessary conditions in Theorem 5.6 are also sufficient. The result is formulated in the following theorem.

Theorem 2.15 [21] Let $A \in \mathcal{I}(2,2)$. Then $\mathcal{F}^{<}(A) \neq \emptyset$ if and only if the $a_{12}<1$ and $a_{22}=1$. If this is the case, then

$$
\begin{aligned}
& \mathcal{F}^{<}(A)=\{\left(x_{1}, x_{2}\right) \in \mathcal{I}(2,2) ; x_{1} \in(0,1), \\
&\left.x_{1}<x_{2} \leq \min \left(1,1+x_{1}-a_{12}\right)\right\}, \\
& \text { if } a_{11}=1,(15) \\
& \mathcal{F}^{<}(A)=\left\{\left(x_{1}, x_{2}\right) \in \mathcal{I}(2,2) ; x_{1} \in\left(0, \min \left(a_{11}, a_{12}\right)\right),\right. \\
&\left.x_{2}=1+x_{1}-a_{12} \leq 1\right\}, \\
& \text { if } a_{11}<1 .(16)
\end{aligned}
$$

Theorem 2.16 [21] Let $A \in \mathcal{I}(n, n)$. If $\max \left\{a_{i j} ; j \in N\right\}=1$ for every $i \in N$, then $\mathcal{F}^{=}(A)=\{(c, c, \ldots, c) ; c \in \mathcal{I}\}$. Otherwise, $\mathcal{F}^{=}(A)=\{(0,0, \ldots, 0)\}$.
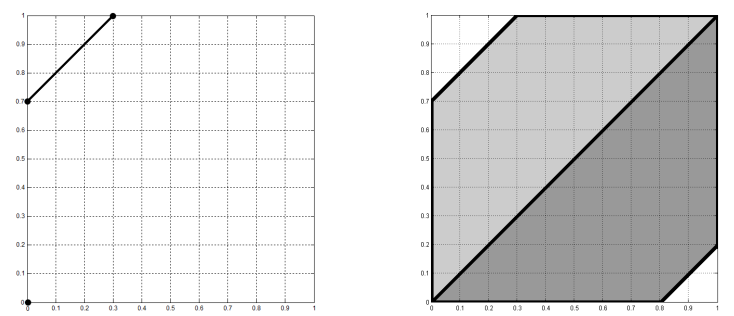

Figure 3: $\mathcal{F}(A)$ and $\mathcal{F}(B)$ in the max-Luk algebra

Example 2.17 Similarly as above, the results are illustrated by computing the eigenspaces of matrices $A, B$ in (1) and (2).

By Theorems 2.15 and 2.16, the eigenspace $\mathcal{F}(A)$ consists of

- increasing eigenvectors of the form $x=$ $\left(x_{1}, 0.7+x_{1}\right)$, with $0 \leq x_{1} \leq 0.3$,

- no decreasing eigenvectors,

- one constant eigenvector $x=(0,0)$.

The eigenspace $\mathcal{F}(B)$ consists of

- increasing eigenvectors with $x_{1}<x_{2} \leq$ $\min \left(0.7+x_{1}, 1\right), 0 \leq x_{1} \leq 1$,

- decreasing eigenvectors with $x_{2}<x_{1} \leq$ $\min \left(0.8+x_{2}, 1\right), 0 \leq x_{2} \leq 1$,

- constant vectors $x=(c, c)$ with $0 \leq c \leq 1$.

Eigenspaces $\mathcal{F}(A), \mathcal{F}(B)$ in max-Luk algebra are shown in Figure 3.

\subsection{Eigenvectors in max-drast algebra}

The drastic norm is the basic example of a nondivisible t-norm on any partially ordered set, see [1]. In this subsection we present the key results from [10]. We work with the max-drast fuzzy algebra $(\mathcal{I}, \oplus, \otimes)$ with binary operation $\oplus=\max$ and $\otimes=$ drast. Hence, for $x, y \in \mathcal{I}(n)$ and $i \in N$ we have

$(x \oplus y)_{i}=\max \left(x_{i}, y_{i}\right)$

$(x \otimes y)_{i}= \begin{cases}\min \left(x_{i}, y_{i}\right) & \text { if } \max \left(x_{i}, y_{i}\right)=1 \\ 0 & \text { if } \max \left(x_{i}, y_{i}\right)<1\end{cases}$

Proposition 2.18 Let $A \in \mathcal{I}(n, n), x \in \mathcal{I}^{<}(n)$. Then $x \in \mathcal{F}^{<}(A)$ if and only if for every $i \in N$ the following hold

$$
\begin{aligned}
a_{i j} & <1 \quad \text { for every } j \in N, j>i \\
a_{i n} & \leq x_{i} \quad \text { if } x_{n}=1
\end{aligned}
$$

$$
\operatorname{drast}\left(a_{i j}, x_{j}\right)=x_{i} \quad \text { for some } j \in\{i, n\}
$$

Theorem 2.19 [10] Let $A \in \mathcal{I}(n, n) . \quad$ Then $\mathcal{F}^{<}(A) \neq \emptyset$ if and only if the following conditions 
are satisfied

$$
\begin{aligned}
& a_{i j}<1 \text { for all } i, j \in N, i<j, \\
& 0<a_{i n} \text { for all } i \in N \backslash\{1\} \text { with } a_{i i}<1, \\
& a_{k n}<a_{\text {in }} \text { for all } i, k \in N, k<i \text { with } a_{i i}<1,
\end{aligned}
$$

$$
a_{n n}=1 \text {. }
$$

Theorem 2.20 [10] Let $A \in \mathcal{I}(n, n)$ fulfill (20)(23) in Theorem 2.19, let $x \in \mathcal{I}^{<}(n)$. Then $x \in$ $\mathcal{F}^{<}(A)$ if and only if the following conditions are satisfied

$$
\begin{gathered}
x_{i}=a_{i n} \text { for all } i \in N \text { with } a_{i i}<1, \\
\text { if } x_{n}=1 \text { then } x_{i} \geq a_{\text {in }} \text { for all } i \in N \backslash\{n\} \\
\text { with } a_{i i}=1, \\
x_{n}=1 \text { if } a_{11}<1,0<a_{1 n}, \\
x_{n}=1 \text { if } a_{i i}<1 \text { for some } i \in N \backslash\{1\} .
\end{gathered}
$$

Theorem $2.21[10]$ Let $A \in \mathcal{I}(n, n)$. If $\max \left\{a_{i j} ; j \in N\right\}=1$ for every $i \in N$, then $\mathcal{F}^{=}(A)=\{(c, c, \ldots, c) ; c \in \mathcal{I}\}$. Otherwise, $\mathcal{F}^{=}(A)=\{(0,0, \ldots, 0)\}$.

Example 2.22 Analogously as in the previous subsections, the results are illustrated by computing the eigenspaces of matrices $A, B$ in (1) and (2).

By Theorems 2.20 and 2.21, the eigenspace $\mathcal{F}(A)$ consists of

- one increasing eigenvector $x=(0.3,1)$,

- no decreasing eigenvectors,

- one constant eigenvector $x=(0,0)$.

The eigenspace $\mathcal{F}(B)$ consists of

- increasing eigenvectors with $x_{1}<x_{2}<1,0 \leq$ $x_{1}<0.3$,

- increasing eigenvectors with $x_{1}<x_{2} \leq 1,0.3 \leq$ $x_{1} \leq 1$

- decreasing eigenvectors with $x_{2}<x_{1}<1,0 \leq$ $x_{2}<0.2$,

- decreasing eigenvectors with $x_{2}<x_{1} \leq 1$, $0.2 \leq x_{2} \leq 1$

- constant vectors $x=(c, c)$ with $0 \leq c \leq 1$.

Eigenspaces $\mathcal{F}(A), \mathcal{F}(B)$ in max-drast algebra are shown in Figure 4.

\section{Robustness in $\max -\min$ algebra}

In this section we shall deal with a transition fuzzy matrix $A$ for which discrete dynamic system is stabilized for every $x$ ( $A$ is called robust) or if the only possibility to arrive at steady state of discrete dynamic system is to start an orbit $x, A \otimes x, A^{2} \otimes x, \ldots$. by a vector that is itself an eigenvector ( $A$ is called weakly robust).

Let $A=\left(a_{i j}\right) \in \mathcal{I}(n, n)$ be a matrix. Let us define the greatest eigenvector $x^{\oplus}(A)$ corresponding to a matrix $A$ as

$$
x^{\oplus}(A)=\bigoplus_{x \in \mathcal{F}(A)} x .
$$
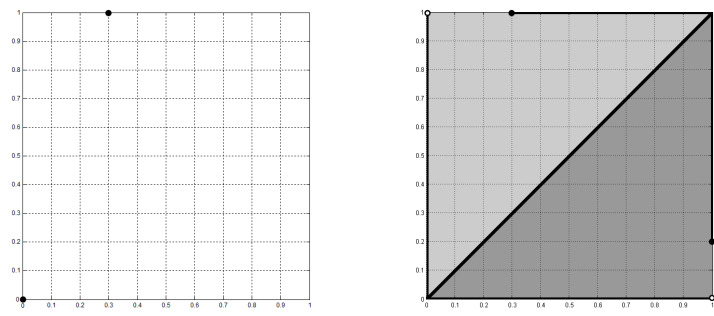

Figure 4: $\mathcal{F}(A)$ and $\mathcal{F}(B)$ in the max-drast algebra

Moreover denote

$$
c(A)=\bigotimes_{i \in N} \bigoplus_{j \in N} a_{i j}, \quad c^{*}(A)=(c(A), \ldots, c(A))^{T} .
$$

It has been proved in [24] for a more general structure (distributive lattice) that the greatest eigenvector $x^{\oplus}(A)$ of $A$ exists for each matrix $A \in \mathcal{I}(n, n)$.

Definition 3.1 For any $A \in \mathcal{I}(n, n)$ and $x \in \mathcal{I}(n)$ the orbit of $A$ generated by $x$ is the vector sequence $\mathcal{O}(A, x)=\left(x(r) ; r \in \mathbb{N}_{0}\right)$ whose initial vector is $x(0)=x$ and successive members are defined by the formula $x(r+1)=A \otimes x(r)$.

Definition 3.2 The sequence $S=(S(r) ; r \in \mathbb{N})$ is ultimately periodic if there is a natural number $p$ such that the following holds for some natural number $R: S(k+p)=S(k)$ for all $k \geq R$. The smallest natural number $p$ with the above property is called the period of $S$, denoted by $\operatorname{per}(S)$.

In the max-min algebra, by max-min operations no new elements are created. Consequently, the matrices in the power sequence of a matrix in max-min algebra only contain the entries from $A$. This implies that the matrix powers sequence is always ultimately periodic. The same holds true for the power sequence $\left(A^{k} ; k \in \mathbb{N}\right)$. Hence a power sequence and an orbit $\mathcal{O}(A, x)$ are always ultimately periodic sequences. Their periods will be called the period of $A$ and the orbit period, in notation $\operatorname{per}(A), \operatorname{per}(A, x)$.

An $O\left(n^{3}\right)$ algorithm for computing $\operatorname{per}(A)$ has been presented in [7] and an $O\left(n^{3} \log n\right)$ algorithm for computing $\operatorname{per}(A, x)$ in $\max -\min$ algebra has been suggested by Semančíková [23].

Let us denote the attraction set and strongly attraction set, by $\operatorname{attr}(A)$ and $\operatorname{attr}^{*}(A)$, respectively as follows

$$
\begin{gathered}
\operatorname{attr}(A)=\{x \in \mathcal{I}(n) ; O(A, x) \cap \mathcal{F}(A) \neq \emptyset\}, \\
\operatorname{attr}^{*}(A)=\left\{x \in \mathcal{I}(n) ; x^{\oplus}(A) \in O(A, x)\right\} .
\end{gathered}
$$

The set $\operatorname{attr}(A)\left(\operatorname{attr}^{*}(A)\right)$ allows us to describe matrices for which an eigenvector (the greatest eigenvector) is reached with any start vector. It is easy to see that $x^{\oplus}(A) \geq c^{*}(A)$ holds true and $x^{\oplus}(A)$ can not be reached with a vector $x \in \mathcal{I}(n), x<c^{*}(A)$.

Let us denote the set $\left\{x \in \mathcal{I}(n) ; x<c^{*}(A)\right\}$ by $M(A)$. 
Definition 3.3 Let $A \in \mathcal{I}(n, n)$ be a matrix. Then $A$ is called

1. weakly robust if $\operatorname{attr}(A)=\mathcal{F}(A)$,

2. robust if $\operatorname{attr}(A)=\mathcal{I}(n)$,

3. strongly robust if $\operatorname{attr}^{*}(A)=\mathcal{I}(n) \backslash M(A)$.

Theorem 3.4 [17] Let $A \in \mathcal{I}(n, n)$ be a matrix. Then $A$ is weakly robust if and only if $\forall x \in$ $\mathcal{I}(n))[A \otimes x \in \mathcal{F}(A) \Rightarrow x \in \mathcal{F}(A)]$.

For a matrix $A \in \mathcal{I}(n, n)$ the symbol $G(A)=$ $(N, E)$ stands for a complete, arc-weighted digraph associated with $A$, i.e., the node set of $G(A)$ is $N$, and the weight of any $\operatorname{arc}(i, j)$ is $a_{i j} \geq O$.

In addition, for given $h \in \mathcal{I}$, the threshold digraph $G(A, h)$ is the digraph with the node set $N$ and with the arc set $E=\left\{(i, j) ; i, j \in N, a_{i j} \geq h\right\}$.

A matrix $A \in \mathcal{I}(n, n)$ is called generalized level$\alpha$ permutation (abrr. level- $\alpha$ permutation) if the threshold digraph $G(A, \alpha)$ is the set of disjoint elementary cycle containing all nodes.

A matrix $A \in \mathcal{I}(n, n)$ is called generalized Hamiltonian permutation if all nonzero entries of $A$ lie on a Hamiltonian cycle (the threshold digraph $G(A, h)$, $h=\min _{i, j \in N}\left\{a_{i j} ; a_{i j}>O\right\}$ is elementary cycle containing all nodes).

Theorem 3.5 [18] Let $A \in \mathcal{I}(n, n)$ be a matrix, $A \neq O$ be a generalized Hamiltonian permutation matrix. Then $A$ is weakly robust if and only if all entries on the Hamiltonian cycle are equal to $I$.

Theorem 3.6 [17] Let $A \in \mathcal{I}(n, n)$ be a matrix. Then $A$ is robust if and only if $\operatorname{per}(A)=1$.

Theorem 3.7 [17] Let $A \in \mathcal{I}(n, n)$ be a matrix. Then $A$ is strongly robust if and only if $x^{\oplus}(A)=$ $c^{*}(A)$ and $G(A, c(A))$ is a strongly connected $d i$ graph with period equal to 1 .

The concepts of strong robustness and robustness has been studied in [17], equivalent conditions and efficient algorithms for interval cases have been presented in [13].

\section{4. $\mathrm{X}$-robustness in $\max -\min$ algebra}

In this section we shall deal with vectors with interval elements. Sufficient and necessary conditions for a matrix to be (weakly) $\mathbf{X}$-robust will be presented.

Definition 4.1 Let $\underline{x}, \bar{x} \in \mathcal{I}(n)$. An interval vector $\mathbf{X}$ with bounds $\underline{x}$ and $\bar{x}$ is defined as follows

$$
\mathbf{X}=[\underline{x}, \bar{x}]=\{x \in \mathcal{I}(n) ; \underline{x} \leq x \leq \bar{x}\} .
$$

Definition 4.2 Let $A \in \mathcal{I}(n, n)$ be a matrix. Then A is called

1. weakly $\mathbf{X}$-robust if $\operatorname{attr}(A) \cap \mathbf{X} \subseteq \mathcal{F}(A)$,

2. $\mathbf{X}$-robust if $\mathbf{X} \subseteq \operatorname{attr}(A)$,

3. strongly $\mathbf{X}$-robust if $\mathbf{X} \subseteq \operatorname{attr}^{*}(A)$.
Let $\mathbf{X}=[\underline{x}, \bar{x}] \in \mathcal{I}(n)$ be an interval vector. For a given index $i \in N$ denote the vector

$$
x^{(i)}=\left(\underline{x}_{1}, \ldots, \underline{x}_{i-1}, \bar{x}_{i}, \underline{x}_{i+1}, \ldots, \underline{x}_{n}\right)^{T} .
$$

Lemma 4.3 For each $x \in \boldsymbol{X}$ there are $\beta_{i} \in \mathcal{I}$ such that $x=\bigoplus_{i=1}^{n} \beta_{i} \otimes x^{(i)}$.

Proof. Let us suppose that $x \in \boldsymbol{X}$. It is easy to see that the assertion follows for $\beta_{i}=x_{i}$.

The next assertions describe a necessary and sufficient conditions for $A$ to be robust.

Lemma 4.4 Let $A$ and $\mathbf{X}$ be given. The following assertions are equivalent

(i) $A$ is $\mathbf{X}$-robust,

(ii) $(\forall x \in \mathbf{X})(\exists k \in \mathbb{N})\left[A^{k} \otimes x \in \mathcal{F}(A)\right]$,

(iii) $(\forall x \in \mathbf{X})[\operatorname{per}(A, x)=1]$.

Theorem 4.5 [14] Let $A$ be a matrix and an interval vector $\mathbf{X}$ be given. A matrix $A$ is $\mathbf{X}$-robust if and only if $\operatorname{per}\left(A, x^{(j)}\right)=1$ for each $j \in N$.

According to the last theorems and the algorithm presented in [23] we compute $\operatorname{per}(A, x)$ and $k_{j}$ by matrix squaring in $O\left(n^{3} \log n\right)$ operations. Then the complexity of an algorithm checking $\mathbf{X}$-robust is $n O\left(n^{3} \log n\right)=O\left(n^{4} \log n\right)$.

\subsection{Weak X-robustness}

We shall define the minimal eigenvector $e_{\underline{x}}$ and maximal eigenvector $f_{\bar{x}}$ from $\mathbf{X}$ which allow to define conditions for a matrix $A$ insuring that each eigenvector $x \in \mathbf{X}, e_{\underline{x}} \leq x \leq f_{\bar{x}}$ is the unique solution of $A \otimes y=x$ in $\mathbf{X}$.

Theorem 4.6 [20] Let $A$ and $\mathbf{X}$ be given. Then $A$ is weakly $\mathbf{X}$-robust if and only if $(\forall x \in \mathbf{X})[A \otimes x \in$ $\mathcal{F}(A) \Rightarrow x \in \mathcal{F}(A)]$.

For every $A \in \mathcal{I}(n, n)$ and $b \in \mathcal{I}(n)$ denote $S(A, b)=\{x \in \mathcal{I}(n) ; A \otimes x=b\}$.

Theorem 4.7 [20] Let $A$ and $\mathbf{X}$ be given. $A$ is weakly $\mathbf{X}$-robust if and only if $(\forall b \in \mathbf{X} \cap$ $\mathcal{F}(A))[|S(A, b) \cap \mathbf{X}|=1]$.

Lemma $4.8[20]$ Let $A=\left(a_{i j}\right) \in \mathcal{I}(n, n)$ be a matrix, $\mathbf{X}=[\underline{x}, \bar{x}] \in \mathcal{I}(n)$ be an interval vector, $\underline{x}<c^{*}(A) \leq \bar{x}$. If $A$ is weakly $\mathbf{X}$-robust then $A$ is level-c(A) permutation. 
For a given level- $c(A)$ permutation matrix $A=$ $\left(a_{i j}\right) \in \mathcal{I}(n, n)$ and $\mathbf{X}=[\underline{x}, \bar{x}] \subseteq \mathcal{I}(n)$ assume that

$$
\begin{aligned}
& \underline{x}<c^{*}(A) \text { and } \max _{i \in N} \underline{x}_{i}<\min _{i \in N} \bar{x}_{i}, \\
& \left(i_{1}, \ldots, i_{n}\right)=\left(i_{1}^{1}, \ldots, i_{s_{1}}^{1}\right) \ldots\left(i_{1}^{k}, \ldots, i_{s_{k}}^{k}\right), \\
& a_{i_{j} i_{j+1}} \geq c(A), 1 \leq j \leq n, \\
& c_{u}=\left(i_{1}^{u}, \ldots, i_{s_{u}}^{u}\right) \text { is an elementary cycle, } \\
& e_{\underline{x}}=\left(e_{1}, \ldots, e_{n}\right)^{T}, \text { where } e_{i}=\max _{v \in c_{u}} \underline{x}_{v}, \\
& f_{\bar{x}}=\left(f_{1}, \ldots, f_{n}\right)^{T}, f_{i}=\min _{v \in c_{u}} \bar{x}_{v} \otimes x_{v}^{\oplus}(A) .
\end{aligned}
$$

We say that $\mathbf{X}$ is invariant under $A$ if $x \in \mathbf{X}$ implies $A \otimes x \in \mathbf{X}$.

Theorem 4.9 [20] Let $\mathbf{X}$ be an invariant under $A$, $A$ be level-c(A) permutation fulfilling (28)-(33) and $\underline{x}<c^{*}(A) \leq \bar{x}$. Then $A$ is weakly $\mathbf{X}$-robust if and only if

$$
\begin{aligned}
& \text { 1. } x_{i_{j+1}}<e_{i_{j+1}} \Rightarrow a_{i_{j} k}<e_{i_{j}} \text { for } k \neq i_{j+1}, k \in N \\
& \text { 2. } \underline{x}_{i_{j+1}}=e_{i_{j+1}} \Rightarrow a_{i_{j} k} \leq e_{i_{j}} \text { for } k \neq i_{j+1}, k \in N \\
& \text { 3. } a_{i_{j} i_{j+1}}=\min _{(k, l) \in c_{u}} a_{k l}=x_{i_{j+1}}^{\oplus}(A)=f_{i_{j+1}} \Rightarrow \\
& \bar{x}_{i_{j+1}} \leq x_{i_{j+1}}^{\oplus}(A) .
\end{aligned}
$$

According to the last theorem the complexity of checking the weakly $\mathbf{X}$-robustness of a given matrix $A$ and an interval vector $\mathbf{X}$ in case that $\underline{x}<$ $c^{*}(A) \leq \bar{x}$ consists of $O\left(n^{2}\right)$ arithmetic operations needed for the checking the condition of $A$ to be a level- $c(A)$ permutation matrix.

\section{Robustness in max-drast algebra}

Let $A \in \mathcal{I}(n, n)$ and $x \in \mathcal{I}(n)$. The orbit $\mathcal{O}(A, x)$ of $x=x(0)$ generated by $A$ in max-drast algebra is defined similarly to $\max -$ min algebra; it is the sequence $x(0), x(1), x(2), \ldots, x(n), \ldots$, where $x(r)=A^{r} \otimes_{d} x(0)$ for each $r \in \mathbb{N}$.

Lemma 5.1 [15] Let $A \in \mathcal{I}(n, n)$ be given. Then $\mathcal{F}(A) \neq \emptyset$.

Similarly as in the $\max -\min$ algebra, by maxdrast operations no new elements (except 0) are created. Consequently, the matrices in the power sequence of a matrix in max-drast algebra only contain the entries from $A$ and 0 . This implies that the matrix powers sequence in max-drast algebra is always ultimately periodic. The same holds true for the power sequence $\left(A^{k} ; k \in \mathbb{N}\right)$. Hence a power sequence and an orbit $\mathcal{O}(A, x)$ are always ultimately periodic sequences.

Notice that an $O\left(n^{3} \log n\right)$ algorithm for computing $\operatorname{per}(A, x)$ in $\max$-min algebra suggested by Semančíková [23] can be easily adapted for computing $\operatorname{per}(A, x)$ in max-drast algebra.

The following theorem gives the formula for computing the matrix period.
Theorem $5.2[9]$ Let $A \in \mathcal{I}(n, n)$. Then

$$
\text { per } A=\operatorname{lcm}\left\{\text { per } \mathcal{K} ; \mathcal{K} \in \mathrm{SCC}^{\star} \mathcal{G}(A, 1)\right\} \text {. }
$$

According to [9] computing the matrix period in max-drast algebra requires $O\left(n^{3}\right)$ arithmetic operations.

Theorem 5.3 [15] Let $A=\left(a_{i j}\right) \in \mathcal{I}(n, n)$. Then $A$ is robust if and only if per $A=1$.

\subsection{X-robustness}

Since we work with $\mathcal{O}(A, x)$ we need to have a condition which guarantees that each element of $\mathcal{O}(A, x)$ is lying in $\boldsymbol{X}$. This is a reason to suppose that $\boldsymbol{X}$ is invariant under $A$ (if and only if $A \otimes_{d} \underline{x} \geq \underline{x}$ and $A \otimes_{d} \bar{x} \leq \bar{x}$ ). From now we suppose that $\boldsymbol{X}$ is invariant under $A$.

At first we will consider the case $\boldsymbol{X}=[\underline{x}, \bar{x}]$ with $\bar{x}=(1, \ldots, 1)^{T}($ denoted $\boldsymbol{X}=[\underline{x}, 1])$.

Lemma 5.4 For each $x \in \boldsymbol{X}=[\underline{x}, 1]$ there are $\beta_{i} \in$ $\mathcal{I}$ such that $x=\bigoplus_{i=1}^{n} \beta_{i} \otimes_{d} x^{(i)}$.

Proof. Let us suppose that $x \in \boldsymbol{X}$. It is easy to see that the assertion follows for $\beta_{i}=x_{i}$.

Theorem 5.5 Let $A \in \mathcal{I}(n, n)$ be a matrix and an interval vector $\boldsymbol{X}=[\underline{x}, 1]$ be given. A matrix $A$ is $\boldsymbol{X}$-robust if and only if $(\forall j \in N)\left[\operatorname{per}\left(A, x^{(j)}\right)=1\right]$.

Let $A \in \mathcal{I}(n, n)$ be given and $\boldsymbol{X}=[\underline{x}, \bar{x}]$ with $0 \leq \underline{x} \leq \bar{x}<(1, \ldots, 1)^{T}$ (abbr. $\left.\boldsymbol{X}=[\underline{x}, \bar{x}], \bar{x}<1\right)$. For $A=\left(a_{i j}\right)$ denote by $\tilde{A}=\left(\tilde{a}_{i j}\right)$ the matrix which arose from $A$ by the following way

$$
\tilde{a}_{i j}= \begin{cases}0, & \text { if } a_{i j}<1 \\ 1, & \text { otherwise }\end{cases}
$$

For a given matrix $A \in \mathcal{I}(n, n)$ denote the eigenspace of $\tilde{A} \in \mathcal{I}(n, n)$ in $\max$-min algebra by $\mathcal{F}(\tilde{A})=\{x \in \mathcal{I}(n) ; A \otimes x=x\}$.

Lemma 5.6 $A \otimes_{d} x=\tilde{A} \otimes x$ and $\mathcal{F}(A)=\mathcal{F}(\tilde{A})$.

Lemma 5.7 Let $A$ and $\boldsymbol{X}=[\underline{x}, \bar{x}], \bar{x}<1$ be given. Then $A$ is $\boldsymbol{X}$-robust in max-drast algebra if and only if $\tilde{A}$ is $\boldsymbol{X}$-robust in max-min algebra.

Proof. By Lemma 5.6 the assertion follows.

Algorithm $\boldsymbol{X}$-robustness

Input: $A, \boldsymbol{X}=[\underline{x}, 1]$ or $\boldsymbol{X}=[\underline{x}, \bar{x}], \bar{x}<1$.

Output: "yes" in variable xr, if $A$ is $\boldsymbol{X}$-robust; "no" in xr, otherwise.

begin

(1) Compute per $A$

(2) If per $A=1$ then xr := "yes", go to end

(3) $k:=1$

(4) If $k=n+1$ then $\mathrm{xr}:=$ "yes"; go to end 
(5) $x:=x^{(k)}$

(6) Compute $\operatorname{per}(A, x)$

(7) If $\operatorname{per}(A, x) \neq 1$ then xr := "no"; go to end

(8) $k:=k+1$; go to Step (4) end

Notice that computing the period of $A$ in step (1) by an algorithm suggested in [9] requires $O\left(n^{3}\right)$ operations. According to the algorithm by Semančíková [23] and its adapting for max-drast algebra we compute $\operatorname{per}(A, x)$ in $O\left(n^{3} \log n\right)$ operations. As the number of repetitions of the loop (4) - (8) is $n$, the complexity of the whole algorithm is $O\left(n^{4} \log n\right)$.

\section{Conclusion}

The paper deals with the description of the eigenspace structure of a given fuzzy matrix in max- $T$ algebra for the following t-norms $T$ : Gödel, Łukasiewicz, product and drastic. The obtained results of the eigenspace structure are visualized by 2dimestional instances. Moreover, as an application of the eigenspace properties, the equivalent conditions for (weak) robustness of matrices in max-min algebra and max-drast algebra are presented, and efficient algorithms for checking the equivalent conditions of interval fuzzy matrices are suggested.

\section{Acknowledgement}

The support of Czech Science Foundation GAČR \#14-02424S and the grant project UHK FIM SPEV \# 2106 is gratefully acknowledged.

\section{References}

[1] J. Casasnovas, G. Mayor, Discrete t-norms and operations on extended multisets. Fuzzy Sets and Systems 159 (2008), 1165-1177.

[2] K. Cechlárová, Eigenvectors in bottleneck algebra, Lin. Algebra Appl. 175 (1992), 63-73.

[3] K. Cechlárová, Efficient computation of the greatest eigenvector in fuzzy algebra, Tatra Mt. Math. Publ. 12 (1997), 73-79.

[4] G. Cohen, D. Dubois, J. P. Quadrat, M. Viot, A linerar-system-theoretic view of discrete event processes and its use for performance evaluation in manufacturing, IEEE Transactions on Automatic Control, AC-30 (1985), 210-220.

[5] R. A. Cuninghame-Green, Describing industrial processes with interference and approximating their steady-state behavior, Oper. Res. Quart. 13 (1962), 95-100.

[6] R. A. Cuninghame-Green, Minimax Algebra and Application, in: Advances in Imaging and Electron Physics 90, (P. W. Hawkes, Ed.). Academic Press, New York, 1995.

[7] M. Gavalec, Computing matrix period in maxmin algebra, Discrete Appl. Math. 75 (1997), 63-70.
[8] M. Gavalec, Monotone eigenspace structure in max-min algebra, Lin. Algebra Appl. 345 (2002), 149-167.

[9] M. Gavalec, Z. Němcová, Matrix period in maxdrast fuzzy algebra. In: Proceedings of 30th International Conference Mathematical Methods in Industry, Karviná, 2012, 213-218.

[10] M. Gavalec, I. Rashid, R. Cimler, Eigenspace structure of a max-drast fuzzy matrix, Fuzzy Sets and Systems (2014), 100-113.

[11] I. Rashid, M. Gavalec, R. Cimler, Eigenspace structure of a max-prod fuzzy matrix, Fuzzy Sets and Systems (to appear).

[12] M. Gondran, M. Minoux, Eigenvalues and eigenvectors in semimodules and their interpretation in graph theory, Proc. 9th Prog. Symp., pp. 133-148, 1976.

[13] M. Molnárová, H. Myšková, J. Plavka, The robustness of interval fuzzy matrices, Linear Algebra and Its Applications 438(8):3350-3364, 2013.

[14] H. Myšková, J. Plavka, The X-robustness of interval fuzzy matrices. Linear Algebra and Its Applications 438 (6): 2757-2769, 2013.

[15] H. Myšková, Robustness of Interval Matrices in max-drast Algebra. In: Mathematical Methods in Economics 2013 : proceedings of the 31st international conference, Jihlava : College of Polytechnics, Czech Republic 642-648, 2013.

[16] G. Olsder, Eigenvalues of dynamic max-min systems, in: Discrete Events Dynamic Systems 1, Kluwer Academic Publishers, 1991, 177-201.

[17] J. Plavka, P. Szabó, On the $\lambda$-robustness of matrices over fuzzy algebra, Discrete Applied Mathematics 159(5):381-388, 2011.

[18] J. Plavka, On the weak robustness of fuzzy matrices, Kybernetika 49(1):128-140, 2013.

[19] J. Plavka, The weak robustness of interval matrices in max-plus algebra, Discrete Applied Mathematics 173:92-101, 2014.

[20] J. Plavka, S. Sergeev, Characterizing matrices with $X$-simple image eigenspace in max-min semiring (2014 submitted to FSS) http://arxiv.org/pdf/1401.3691.pdf.

[21] I. Rashid, M. Gavalec, S. Sergeev, Eigenspace of a three-dimensional max-Lukasiewicz fuzzy matrix, Kybernetika, 48 (2012), 309-328.

[22] E. Sanchez, Resolution of eigen fuzzy sets equations, Fuzzy Sets and Systems 1 (1978), 69-74.

[23] B. Semančíková, Orbits in max-min algebra, Linear Algebra Appl. 414 (2006) 38-63.

[24] Yi-Jia Tan, Eigenvalues and eigenvectors for matrices over distributive lattices, Lin. Algebra Appl. 283 (1998), 257-272. 